\title{
BIFREE OBJECTS IN $e$-VARIETIES OF STRICT ORTHODOX SEMIGROUPS AND THE LATTICE OF STRICT ORTHODOX *-SEMIGROUP VARIETIES
}

\author{
by KARL AUINGER
}

(Received 17 June, 1991)

1. Introduction. For regular semigroups, the appropriate analogue of the concept of a variety seems to be that of an $e$ (xistence)-variety, developed by Hall $[6,7,8]$. A class $\mathscr{V}$ of regular semigroups is an $e$-variety if it is closed under taking direct products, regular subsemigroups and homomorphic images. For orthodox semigroups, this concept has been introduced under the term "bivariety" by Kadourek and Szendrei [12]. Hall showed that the collection of all $e$-varieties of regular semigroups forms a complete lattice under inclusion. Further, he proved a Birkhoff-type theorem: each $e$-variety is determined by a set of identities. For $e$-varieties of orthodox semigroups a similar result has been proved by Kadourek and Szendrei. At variance with the case of varieties, prima facie the free objects in general do not exist for $e$-varieties. For instance, there is no free regular or free orthodox semigroup. This seems to be true for most of the naturally appearing $e$-varieties (except for cases of $e$-varieties which coincide with varieties of unary semigroups such as the classes of all inverse and completely regular semigroups, respectively). This is true if the underlying concept of free objects is defined as usual. Kadourek and Szendrei adopted the definition of a free object according to $e$-varieties of orthodox semigroups by taking into account generalized inverses in an appropriate way. They called such semigroups bifree objects. These semigroups satisfy the properties one intuitively expects from the "most general members" of a given class of semigroups. In particular, each semigroup in the given class is a homomorphic image of a bifree object, provided the bifree objects exist on sets of any cardinality. Concerning existence, Kadourek and Szendrei were able to prove that in any class of orthodox semigroups which is closed under taking direct products and regular subsemigroups, all bifree objects exist and are unique up to isomorphism. Further, similar to the case of varieties, there is an order inverting bijection between the fully invariant congruences on the bifree orthodox semigroup on an infinite set and the $e$-varieties of orthodox semigroups. Recently, Y. T. Yeh [22] has shown that suitable analogues to free objects exist in an $e$-variety $\mathcal{V}$ of regular semigroups if and only if all members of $\mathscr{V}$ are either $E$-solid or locally inverse.

The purpose of the present paper is to construct concrete realizations of the bifree objects in certain $e$-varieties of strict orthodox semigroups. This will be done in Section 3 after having introduced several definitions and results in Section 2. The method of description of the respective bifree objects is the same as used by the author for the description of free strict inverse semigroups [2,3]. Several of the so obtained bifree objects can be interpreted as the relatively free objects in certain varieties of strict orthodox *-semigroups. This motivates the study of the lattice of strict orthodox *-semigroup varieties which will be done in Section 4. This lattice will be completely described modulo the lattice of all group varieties. It turns out that each relatively free strict orthodox $*$-semigroup is the bifree object in some $e$-variety whereas the converse does not hold. 
2. Preliminaries. For all undefined notions and unproved statements not explicitly marked, the reader is referred to the books of Howie [11] and Petrich [18] (semigroups), Grätzer [4, 5] (universal algebras, lattices) and Harary [10] (graphs).

A semigroup $S$ is strict regular if it is a regular subdirect product of completely 0 -simple and/or completely simple semigroups. These semigroups have been studied extensively by Lallement and Petrich $[12,13,17]$. Some recent results can be found in the papers of Hall $[6,7]$. The author $[2,3]$ studied free objects in certain unary semigroup varieties all of whose members are strict regular semigroups. Similarly as for the inverse case (see [18, Chap. XII]), $e$-varieties of strict regular semigroups form the "bottom" of the lattice of all $e$-varieties of regular semigroups. By some reformulation of a result of Petrich [17, Theorem 3.4] the following can be obtained (see [3, Theorem 2.3]).

THEOREM 2.1. Let $X$ be a partially ordered set. For all $\alpha \in X$ let $I_{\alpha}$ denote completely 0 -simple semigroups whose non-zero parts $I_{\alpha}^{*}$ are disjoint for distinct $\alpha, \beta$. For each pair $\alpha \geq \beta \in X$ let $f_{\alpha, \beta}: I_{\alpha}^{*} \rightarrow I_{\beta}^{*}$ be a partial homomorphism subject to the following conditions:

(1) $f_{\alpha, \alpha}=\operatorname{id}_{l_{\alpha}^{*}}$ for all $\alpha \in X$,

(2) $f_{\alpha, \beta} f_{\beta, \gamma}=f_{\alpha, \gamma}$ whenever $\alpha \geq \beta \geq \gamma$,

(3) for any $a \in I_{\alpha}^{*}, b \in I_{\beta}^{*}$ the set

$$
D(a, b)=\left\{\gamma \leq \alpha, \beta \mid\left(a f_{\alpha, \gamma}\right)\left(b f_{\beta, \gamma}\right) \neq 0 \text { in } I_{\gamma}\right\}
$$

has a greatest element, to be denoted by $\delta(a, b)$.

On $S=\bigcup_{\alpha \in X} I_{\alpha}^{*}$ define a multiplication by

$$
a b=\left(a f_{\alpha, \delta(a, b)}\right)\left(b f_{\beta, \delta(a, b)}\right)
$$

where $a \in I_{\alpha}^{*}, b \in I_{\beta}^{*}$ and the product is computed in $I_{\delta(a, b)}^{*}$. Then the groupoid $S$, to be denoted by $\left(X ; I_{\alpha}, f_{\alpha, \beta}\right)$, is a strict regular semigroup. Conversely, every strict regular semigroup can be so constructed.

As a consequence of this description, each strict regular semigroup is a subdirect product of its principal factors.

As already mentioned, a class $\mathscr{V}$ of regular semigroups which is closed under taking direct products, regular subsemigroups and homomorphic images is an e(xistence)-variety of regular semigroups. The following concepts have been introduced by Kadourek and Szendrei [12]. Let $I$ be a non-empty set, $I^{*}=\left\{i^{*} \mid i \in I\right\}$ be a disjoint copy of $I$ with $i \mapsto i^{*}$ being a bijection. Let $\mathscr{V}$ be a class of orthodox semigroups. The bifree object in $\mathscr{V}$ on $I$ is a pair $(S, \phi)$ where $S \in \mathscr{V}$ and $\phi: I \cup I^{*} \rightarrow S$ is a mapping such that $i^{*} \phi$ is an inverse of $i \phi$ for each $i \in I$ which satisfies the following universal property. For any $T \in \mathscr{V}$ and any mapping $\psi: I \cup I^{*} \rightarrow T$ such that $i^{*} \psi$ is an inverse of $i \psi$ for each $i \in I$, there exists a unique homomorphism $\bar{\psi}: S \rightarrow T$ which extends $\psi$, that is, satisfying $\phi \bar{\psi}=\psi$. In each class of orthodox semigroups there is, up to isomorphism, at most one bifree object on a non-empty set $I$. Concerning existence, Kadourek and Szendrei have proved the following.

THEOREM 2.2. In any class $\mathscr{V}$ of orthodox semigroups which is closed under taking regular subsemigroups and direct products, the bifree object exists on any non-empty set $I$. This semigroup, to be denoted by $F \mathcal{V}_{I}$, is generated by $\left(I \cup I^{*}\right) \phi$.

By the following result, also proved by Kadourek and Szendrei, the particular nearness to the theory of varieties can be observed. 
THEOREM 2.3. Let FO, be the bifree orthodox semigroup on an infinite set $I$. There is an order inverting bijection between the lattice of all fully invariant congruences on $F O_{I}$ and the lattice of all e-varieties of orthodox semigroups. Further, if $\rho_{V}$ is the fully invariant congruence corresponding to the e-variety $\mathscr{V}$ then the relatively bifree object in $\mathscr{V}$ is given by $F \mathscr{V}_{1} \cong F \mathcal{O}_{I} / \rho_{V}$. The congruence $\rho_{V}$ is determined by all "identities" which hold in $\mathscr{V}$.

The precise definition of "an identity holds in an orthodox semigroup $S$ " is given in Section 3. The bifree object $F O_{1}$ is isomorphic to the free orthodox *-semigroup $F O_{1}^{*}$ which has been described by Szendrei [20,21]. By Nordahl and Scheiblich [16], a semigroup $S$, endowed with a unary operation $s \mapsto s^{*}$ is a regular *-semigroup if the following laws are satisfied:

(1) $(x y)^{*}=y^{*} x^{*}$,

(2) $\left(x^{*}\right)^{*}=x$,

(3) $x x^{*} x=x$.

An operation * satisfying (1) and (2) is an involution. Nordahl and Scheiblich showed the following.

THeOREM 2.4. A regular *-semigroup is orthodox if and only if it satisfies the identity

(4) $x x^{*} y y^{*} z z^{*}=\left(x x^{*} y y^{*} z z^{*}\right)^{2}$.

Consequently, the class of all orthodox *-semigroups forms a subvariety of the variety of all regular *-semigroups.

We call a regular (orthodox) *-semigroups which is in addition strict a strict regular (orthodox) *-semigroup. Finally we give some more definitions. A regular semigroup $S$ is $\mathscr{R} / \mathscr{L}$-unipotent if each $\mathscr{R} / \mathscr{L}$-class of $S$ contains only one idempotent; $S$ is combinatorial if $\mathscr{H}$ is the identical relation, or equivalently, all subgroups are trivial. We finish the present section by giving a list of symbols which will be used in the following. In general, $e$-varieties will be denoted by script letters $\mathscr{V}$ whereas $\mathscr{V}^{*}$ is the variety of regular *-semigroups all of whose members belong to $\mathscr{V}$. For instance, $\mathscr{R} \mathscr{B}$ is the class of all rectangular bands and $\mathscr{R} \mathscr{B}^{*}$ denotes the class of all rectangular bands which are equipped with an involution. These classes do not coincide and not even the respective classes of underlying semigroups do. On the other hand, if $\mathscr{V}$ is an $e$-variety consisting entirely of inverse semigroups then $\mathscr{V}$ and $\mathscr{V}^{*}$ can be identified by the mutual inverse mappings $(S, \cdot) \mapsto\left(S, \cdot{ }^{*}\right),\left(S, \cdot,{ }^{*}\right) \mapsto(S, \cdot)$ since the involution ${ }^{*}$ is uniquely determined in each inverse semigroup $(S, \cdot)$.

0 the class of all orthodox semigroups

$\mathscr{I}$ the class of all inverse semigroups

$\mathscr{S \mathscr { R }}$ the class of all strict regular semigroups

90 the class of all strict orthodox semigroups

$\mathscr{S}$ the class of all strict inverse semigroups

$\mathscr{T}$ the class of trivial semigroups

$\mathscr{G}$ the class of all groups

$\mathscr{S}$ the class of all semilattices 


$\begin{aligned} \mathscr{R} \mathscr{B} & \text { the class of all rectangular bands } \\ \mathscr{B} & \text { the class of all combinatorial strict inverse semigroups } \\ \mathscr{R} \mathscr{Z} & \text { the class of all right zero semigroups } \\ \mathscr{L} \mathscr{Z} & \text { the class of all left zero semigroups } \\ \mathscr{S} \mathscr{R} \mathscr{U} & \text { the class of all strict } \mathscr{R} \text {-unipotent semigroups } \\ \mathscr{S} \mathscr{L} U & \text { the class of all strict } \mathscr{L} \text {-unipotent semigroups } \\ \mathcal{N} \mathscr{B} & \text { the class of all normal bands } \\ \mathscr{L} \mathscr{N} \mathscr{B} & \text { the class of all left normal bands } \\ \mathscr{R} \mathcal{R} \mathscr{B} & \text { the class of all right normal bands } \\ \mathscr{C V} & \text { the combinatorial members of } \mathscr{V} \\ \mathscr{L}(\mathscr{V}) & \text { the lattice of all sub-e-varieties of the } e \text {-variety } \mathscr{V} \\ \mathscr{L}(\mathscr{V} *) & \text { the lattice of all subvarieties of } \mathscr{V}^{*} \\ F \mathscr{V} & \text { the bifree object in the } e \text {-variety } \mathscr{V} \text { on } I \\ F_{I}^{*} & \text { the free object in the variety } \mathscr{V}^{*} \text { on } I \\ \langle S\rangle & \text { the } e \text {-variety generated by } S \\ \langle S\rangle^{*} & \text { the variety generated by the involutorial semigroup } S \\ C_{2} & \text { the five element non-orthodox } 0 \text {-simple semigroup } \\ \varepsilon_{I} & \text { the identical relation on } I \\ \omega_{I} & \text { the universal relation on } I \\ E(A) & \text { the set of all idempotents of } A \subseteq S \\ V(x) & \text { the set of inverses of the element } x\end{aligned}$

3. Bifree objects in e-varieties of strict orthodox semigroups. In this section we first shall consider the lattice of $e$-varieties of strict orthodox semigroups. Then we shall derive models of the bifree objects in certain of the $e$-varieties under study. This also will lead to solutions of the word problem for the bifree objects in the respective $e$-varieties. The next two results are essentially contained in the papers of Hall $[\mathbf{7 , 8}]$.

THEOREM 3.1. Let $\mathscr{A}$ be an e-variety of groups and $\mathscr{S O}(\mathscr{A})$ denote the e-variety of all strict orthodox semigroups all of whose subgroups belong to $\mathscr{A}$. Then $\mathscr{S O}(\mathscr{A})=\mathscr{B} \vee \mathscr{A} \vee$ $\mathscr{R} \mathscr{B}$. In particular, $\mathscr{S O}=\mathscr{B} \vee \mathscr{G} \vee \mathscr{R} \mathscr{B}$ and $\mathscr{C} \mathscr{Y} O=\mathscr{B} \vee \mathscr{R} \mathscr{B}$.

THEOREM 3.2. The lattice of all e-varieties of combinatorial strict regular semigroups is as shown in Figure 1.

Remark. Notice that the combinatorial strict right (left) unipotent semigroups are precisely the combinatorial strict left (right) generalized inverse semigroups. By [7, Theorem 4.4] it follows that $\mathscr{L}(\mathscr{S O}) \cong \mathscr{L}(\mathscr{C} \mathscr{Y O}) \times \mathscr{L}(\mathscr{G})$. Therefore we have a description of the lattice $\mathscr{L}(\mathscr{S O})$ modulo the lattice of all group varieties $\mathscr{L}(\mathscr{G})$. Further, each of the $e$-varieties of combinatorial strict regular semigroups is generated by a single member. 


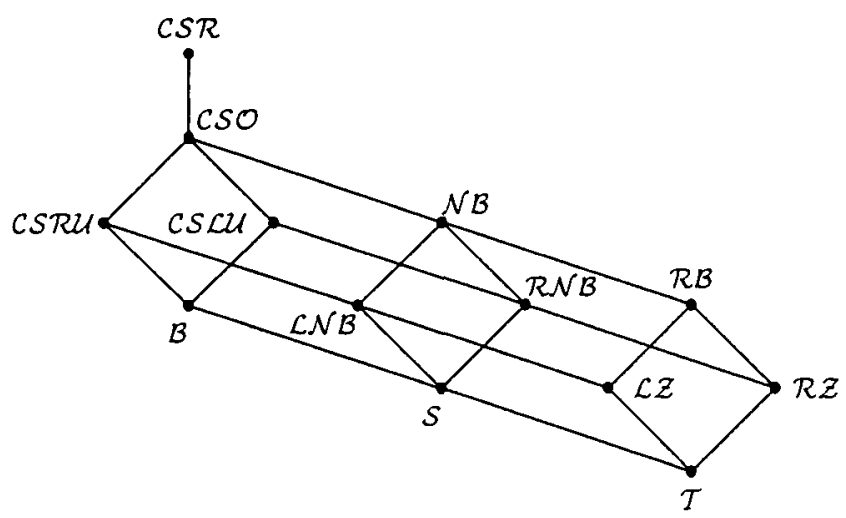

Figure 1

We now consider the bifree objects in orthodox $e$-varieties of strict regular semigroups. Let $F O_{I}$ be the bifree orthodox semigroup on $I$ for an infinite set $I$. Let $\rho_{\mathscr{B}}$ and $\rho_{\mathscr{R} \mathscr{B}}$ denote the fully invariant congruences on $F O_{I}$ associated with the $e$-varieties $\mathscr{B}$ and $\mathscr{R} \mathscr{B}$, respectively. These fully invariant congruences are determined by the "biidentities" which hold in the respective $e$-varieties (for details see Kadourek and Szendrei [12]). By Theorems 2.2 and $2.3, \rho_{\mathscr{B} \vee \mathscr{R} \mathscr{B}}=\rho_{\mathscr{B}} \cap \rho_{\mathscr{R} \mathscr{B}}$. In particular, $F(\mathscr{B} \vee \mathscr{R} \mathscr{B})_{I}$ is isomorphic to the subdirect product of $F \mathscr{B}_{I} \times F \mathscr{R} \mathscr{B}_{I}=F \mathscr{O}_{I} / \rho_{\mathscr{B}} \times F O_{I} / \rho_{\mathscr{R} \mathscr{B}}$ which is generated by all pairs

$$
\left\{\left(i \rho_{\mathscr{B}}, i \rho_{\mathscr{R} \mathscr{B}}\right), \quad\left(i^{*} \rho_{\mathscr{B}}, i^{*} \rho_{\mathscr{R} \mathscr{B}}\right) \mid i \in I\right\}
$$

Here $I \cup I^{*}$ is considered as a subset of $F O_{l}$. A model of $F(\mathscr{B} \vee \mathscr{R} \mathscr{B})_{I}$ now can be determined in terms of $F \mathscr{B}_{I}$ and $F \mathscr{R} \mathscr{B}_{I}$. Since the bifree objects are uniquely determined up to isomorphism, the following construction also applies for finite sets $I$. Further, since in a rectangular band any two elements are mutual inverses, the bifree rectangular band on $I$ can be shown to be the free rectangular band on $I \cup I^{*}$. This is the rectingular band on $\left(I \cup I^{*}\right) \times\left(I \cup I^{*}\right)$ which is freely generated by $\left\{(i, i),\left(i^{*}, i^{*}\right) \mid i \in I\right\}$. For convenience of the further development, we give a slightly different model of the bifree rectangular band on $I$.

LeMMA 3.3. Let $I$ be a non-empty set and $R_{I}=\left\{r_{i} \mid i \in I\right\}$ and $L_{I}=\left\{l_{i} \mid i \in I\right\}$ be two disjoint sets of cardinality $|I|$. Let $Y=R_{I} \cup L_{l}$ and $\phi: i \mapsto\left(l_{i}, r_{i}\right), i^{*} \mapsto\left(r_{i}, l_{i}\right)$. Then $(Y \times Y, \phi)$ is the bifree rectangular band on $I$.

Proof. The mapping $\phi$ can be uniquely extended to a homomorphism of the free rectangular band on $I \cup I^{*}$ into the rectangular band $Y \times Y$. Since $\left(I \cup I^{*}\right) \phi$ generates $Y \times Y$, this extension is onto. Further, it is routine to verify that the extension of $\phi$ is one-to-one.

Let a unary operation * on $Y \times Y$ be defined by $(x, y)^{*}=(y, x)$; then $\left(Y \times Y, \cdot,^{*}\right)$ is the free rectangular *-band, freely generated by $\left\{\left(l_{i}, r_{i}\right) \mid i \in I\right\}$. In order to present a model of $F \mathscr{B}_{I}$ we need the following definitions. 
Definition 1 ([2]). Let $I$ be a non-empty set, $R_{I}=\left\{r_{i} \mid i \in I\right\}$ and $L_{I}=\left\{l_{i} \mid i \in I\right\}$ be two disjoint sets of cardinality $|I|$. Let $\mathscr{P} I$ denote the set of all finite non-empty subsets of I. For $A \in \mathscr{P I}$ let $Y_{A}=\left\{l_{i}, r_{i} \mid i \in A\right\}$ and $Y=L_{I} \cup R_{l}$. For $Z \subseteq Y$ let $c(Z)=\left\{i \in I \mid l_{i} \in Z\right.$ or $\left.r_{i} \in Z\right\}$ and $c\left(l_{i}\right)=c\left(r_{i}\right)=i$ for all $i \in I$. An equivalence relation $\alpha$ on $Y_{A}$ is admissible if for any $x, y \in Y_{A}$ there exist $x_{i_{1}}, y_{i_{1}}, \ldots, x_{i_{n}}, y_{i_{n}} \in Y_{A}, x_{i_{k}}, y_{i_{k}} \in\left\{l_{i_{k}}, r_{i_{k}}\right\}$, such that

$$
x \propto x_{i_{1}}, y_{i_{1}} \alpha x_{i_{2}}, \ldots, y_{i_{n}} \alpha y .
$$

Let $X_{A}$ be the set of all admissible equivalence relations $\alpha$ on $Y_{A}$. Further, let $X=\bigcup_{A \in \mathscr{P} I} X_{A}$. For each $\alpha \in X$ let $d \alpha=\{x \in Y \mid x \alpha x\}$ and $Y / \alpha=\{x \alpha \mid x \in Y\}=d \alpha / \alpha$.

In [2] the following has been proved about $F \mathscr{B}$.

TheOREM 3.4. Let $Y, X, Y / \alpha$ etc. be as in Definition 1 and

$$
S=\bigcup_{\alpha \in X} Y / \alpha \times\{\alpha\} \times Y / \alpha
$$

endowed with multiplication

$$
(x \alpha, \alpha, y \alpha)(u \beta, \beta, v \beta)=(x \delta, \delta, v \delta)
$$

where $\delta=\delta(\alpha, y, u, \beta)=\alpha \vee \omega_{\{y, u\}} \vee \beta$ and the join is taken in the lattice of all symmetric and transitive relations on $Y$, and also endowed with inversion

$$
(x \alpha, \alpha, y \alpha)^{-1}=(y \alpha, \alpha, x \alpha) .
$$

Then $\left(S, \cdot,^{-1}\right)$ is the free object in the unary semigroup variety $\mathscr{B}$, with free generators $\left\{\left(l_{i} \varepsilon_{i}, \varepsilon_{i}, r_{i} \varepsilon_{i}\right) \mid i \in I\right\}$ where $\varepsilon_{i}=\varepsilon_{\left\{l_{i}, r_{i}\right\}}$.

Different interpretations of $F \mathscr{B}$, have been obtained by Reilly [19] by means of "strict graphs" and by Margolis, Meakin and Stephen [15] by means of "inverse automata".

For convenience of notation, the elements of $F \mathscr{B}_{I} \times F \mathscr{R} \mathscr{B}_{l}$ will be realized as follows:

$$
F \mathscr{B}_{I} \times F \mathscr{R} \mathscr{B}_{l}=\left\{(u, x \alpha, \alpha, y \alpha, v) \mid(x \alpha, \alpha, y \alpha) \in F \mathscr{B}_{l},(u, v) \in Y \times Y\right\},
$$

endowed with multiplication

$$
(u, x \alpha, \alpha, y \alpha, v)(a, c \beta, \beta, d \beta, b)=(u, x \delta, \delta, d \delta, b)
$$

where $\delta=\delta(\alpha, y, c, \beta)=\alpha \vee \omega_{\{y, c\}} \vee \beta$. Denote by $S$ the subsemigroup of $F \mathscr{B}_{I} \times F \mathscr{R}_{1}$ which is generated by the set

$$
\left\{\left(l_{i}, l_{i} \varepsilon_{i}, \varepsilon_{i}, r_{i} \varepsilon_{i}, r_{i}\right),\left(r_{i}, r_{i} \varepsilon_{i}, \varepsilon_{i}, l_{i} \varepsilon_{i}, l_{i}\right) \mid i \in I\right\} .
$$

Let $\alpha \in X$ and $x, y \in d \alpha$. Then $(x \alpha, \alpha, y \alpha) \in F \mathscr{B}_{1}$. Since $F \mathscr{B}_{1}$ is generated as a semigroup by the elements

$$
\left\{\left(l_{i} \varepsilon_{i}, \varepsilon_{i}, r_{i} \varepsilon_{i}\right),\left(r_{i} \varepsilon_{i}, \varepsilon_{i}, l_{i} \varepsilon_{i}\right) \mid i \in I\right\}
$$

there are $u \in x \alpha, v \in y \alpha$ such that $(u, x \alpha, \alpha, y \alpha, v) \in S$. Since the elements $\left(x, x \varepsilon_{i}, \varepsilon_{i}, x \varepsilon_{i}, x\right)$ and $\left(y, y \varepsilon_{j}, \varepsilon_{j}, y \varepsilon_{j}, y\right)$ are contained in $S$ provided $c(x)=i$ and $c(y)=j$, by multiplication from the left and the right we get $(x, x \alpha, \alpha, y \alpha, y) \in S$. On the other hand, suppose that $(u, x \alpha, \alpha, y \alpha, v) \in S$. This element is a product of elements of the form $\left(l_{i}, l_{i} \varepsilon_{i}, \varepsilon_{i}, r_{i} \varepsilon_{i}, r_{i}\right)$ and $\left(r_{i}, r_{i} \varepsilon_{i}, \varepsilon_{i}, l_{i} \varepsilon_{i}, l_{i}\right)$ so that $u, v \in d \alpha$ and $u \alpha x, v \alpha y$. 
Consequently,

$$
S=\{(x, x \alpha, \alpha, y \alpha, y) \mid \alpha \in X, x, y \in d \alpha\} .
$$

The entries $x \alpha, y \alpha$ are redundant and may be omitted. In other words, the mapping $(x, x \alpha, \alpha, y \alpha, y) \mapsto(x, \alpha, y)$ is a bijection between $S$ and $\bigcup_{A \in \mathscr{P}_{1}} Y_{A} \times X_{A} \times Y_{A}$. We thus have obtained the following model of the bifree combinatorial strict orthodox semigroup $F \mathscr{C} \mathscr{S O}$.

THEOREM 3.5. Let I be a non-empty set and $\mathscr{P I}, Y_{A}, X_{A}$ etc. be as in Definition 1. Let $S=\bigcup_{A \in \mathscr{P} I} Y_{A} \times X_{A} \times Y_{A}$, endowed with multiplication

$$
(x, \alpha, y)(u, \beta, v)=\left(x, \alpha \vee \omega_{\{y, u\}} \vee \beta, v\right) .
$$

Let $\phi: i \mapsto\left(l_{i}, \varepsilon_{i}, r_{i}\right), i^{*} \mapsto\left(r_{i}, \varepsilon_{i}, l_{i}\right)$. Then $(S, \phi)$ is the bifree combinatorial strict orthodox semigroup on $I$.

Using the fact that the bifree left/right zero semigroup on $I$ is the left/right zero semigroup on $I \cup I^{*}$, the same procedure as above yields models of the bifree objects in the $e$-varieties of all strict combinatorial $\mathscr{L}$-respectively $\mathscr{R}$-unipotent semigroups.

Theorem 3.6. Let $I$ be a non-empty set and $Y, X$ etc. be as in Definition 1. Let $S=\cup_{\alpha \in X} d \alpha \times\{\alpha\} \times d \alpha / \alpha$, endowed with multiplication

$$
(x, \alpha, y \alpha)(u, \beta, v \beta)=(x, \delta, v \delta)
$$

where $\delta=\alpha \vee \omega_{\{y, u\}} \vee \beta$. Let $\phi: i \mapsto\left(l_{i}, \varepsilon_{i}, r_{i} \varepsilon_{i}\right), i^{*} \mapsto\left(r_{i}, \varepsilon_{i}, l_{i} \varepsilon_{i}\right)$. Then $(S, \phi)$ is the bifree object on $I$ in the e-variety $\mathscr{C} \mathscr{Y} \mathscr{R} U=\mathscr{B} \vee \mathscr{L} \mathscr{Z}$. The bifree object $F \mathscr{C} \mathscr{Y} \mathscr{L} U_{l}$ is constructed dually.

For $\mathscr{A} \in\{\mathscr{L} \mathscr{Z}, \mathscr{R} \mathscr{Z}, \mathscr{R} \mathscr{B}\}$ let $\mathscr{A}^{-1}$ denote the unary semigroup variety consisting of all semigroups of $\mathscr{A}$ which in addition are equipped with the unary operation $x^{-1}=x$. Let $\mathscr{B}^{-1}$ denote the variety of all combinatorial strict inverse (unary) semigroups. With respect to this notation, the free objects in the unary semigroup varieties $\mathscr{A}^{-1} \vee \mathscr{B}^{-1}$ have been constructed by the author in [3].

The following important definition is due to Kadourek and Szendrei [12]. Let $w=x_{1} \ldots x_{n}$ and $v=y_{1} \ldots y_{m}$ be two words in the alphabet $I \cup I^{*}$. Let $S$ be an orthodox semigroup. Then the identity $w=v$ holds in $S$ if for any mapping $\psi: I \cup I^{*} \rightarrow S$ such that $i^{*} \psi \in V(i \psi)$ for all $i \in I, x_{1} \psi \ldots x_{n} \psi=y_{1} \psi \ldots y_{m} \psi$. The identity $w=v$ holds in the class $\mathscr{V}$ of orthodox semigroups if it holds in each member of $\mathscr{V}$.

Denote by $\phi$ the mapping $I \cup I^{*} \rightarrow F \mathscr{C} \mathscr{Y O}$, mentioned in Theorem 3.5. If $\left(u_{1}, \varepsilon_{1}, v_{1}\right), \ldots,\left(u_{n}, \varepsilon_{n}, v_{n}\right) \in\left(I \cup I^{*}\right) \phi$ then

$$
\left(u_{1}, \varepsilon_{1}, v_{1}\right) \ldots\left(u_{n}, \varepsilon_{n}, v_{n}\right)=\left(u_{1}, \alpha, v_{n}\right)
$$

where $\alpha$ is the equivalence relation on $\left\{u_{1}, v_{1}, \ldots, u_{n}, v_{n}\right\}$ which is generated by the pairs $\left\{\left(v_{1}, u_{2}\right), \ldots,\left(v_{n-1}, u_{n}\right)\right\}$. Similarly as in [2, section 4] we now may identify $\left(l_{i}, \varepsilon_{i}, r_{i}\right)$ with $\left(i, \varepsilon_{i}, i^{*}\right)$ and $\left(r_{i}, \varepsilon_{i}, l_{i}\right)$ with $\left(i^{*}, \varepsilon_{i}, i\right)$. This yields the following solutions to the problem of deciding which biidentities hold in the $e$-varieties $\mathscr{C} \mathscr{S}, \mathscr{C} \mathscr{Y} \mathscr{L} U, \mathscr{C} \mathscr{Y} \mathscr{R} U$, respectively (see also [2, Theorem 4.1]). In the following, $x^{*}=y \in I$ whenever $x=y^{*} \in I^{*}$.

THEOREM 3.7. Let $I$ be a non-empty set and $w=x_{1} \ldots x_{n}$ and $v=y_{1} \ldots y_{m}$ be two words in the alphabet $I \cup I^{*}$. Let $\alpha$ denote the equivalence relation on $\left\{x_{1}, x_{1}^{*}, \ldots, x_{n}, x_{n}^{*}\right\}$ 
which is generated by the pairs $\left\{\left(x_{1}^{*}, x_{2}\right), \ldots,\left(x_{n-1}^{*}, x_{n}\right)\right\}$ and let $\beta$ be defined analogously for the word $v$. Then the identity $w=v$ holds in $\mathscr{C} \mathscr{S O}(\mathscr{C} \mathscr{R} \mathcal{R}, \mathscr{C} \mathscr{Y} \mathscr{U})$ if and only if

(1) $\alpha=\beta$

(2) $x_{1}=y_{1}\left(x_{1}=y_{1}, x_{1} \alpha=y_{1} \alpha\right)$,

(3) $x_{n}=y_{m}\left(x_{n}^{*} \alpha=y_{m}^{*} \alpha, x_{n}=y_{m}\right)$.

Notice that in (3) we have written $x_{n}=y_{m}$ instead of $x_{n}^{*}=y_{m}^{*}$ since these conditions are equivalent. However, $x_{n} \alpha=y_{m} \alpha$ is not equivalent to $x_{n}^{*} \alpha=y_{m}^{*} \alpha$.

Finally we shall give a model of the bifree object $F \mathscr{S O} \mathcal{O}_{l}$ which arises from $F \mathscr{S} \mathscr{I}_{I}$ and $F \mathscr{R} \mathscr{B}_{1}$ similarly as $F \mathscr{C} \mathscr{Y O} \mathscr{O}_{1}$ arises from $F \mathscr{B}_{1}$ and $F \mathscr{R} \mathscr{B}_{1}$. Since $\mathscr{S} \mathscr{I}=\mathscr{R} \vee \mathscr{G}, F \mathscr{Y} \mathscr{I}_{1}$ is a subdirect product of $F \mathscr{B}_{l}$ and $F \mathscr{G}_{l}$. We first present a description of $F \mathscr{Y} \mathscr{I}_{l}$.

Definition 2 ([3]). Let $I$ be a non-empty set and $Y, X$ etc. be as in Definition 1 . The directed edge-labelled pseudograph $\operatorname{dg}(\alpha)$ associated with $\alpha$ is defined to be the following:

$$
\begin{gathered}
V(d g(\alpha))=Y / \alpha, \\
E(d g(\alpha))=\left\{\left(\left(l_{i} \alpha, r_{i} \alpha\right), i\right),\left(\left(r_{i} \alpha, l_{i} \alpha\right), i^{-1}\right) \mid i \in c(d \alpha)\right\} .
\end{gathered}
$$

That is, the vertices of $d g(\alpha)$ are the equivalence classes modulo $\alpha$ and the directed edge (having label) $i$ starts at $l_{i} \alpha$ and ends at $r_{i} \alpha$ and conversely for $i^{-1}$. With each di-edge $i^{\varepsilon}, \varepsilon \in\{1,-1\}$, we now associate two vertices of $d g(\alpha)$, the starting vertex $s\left(i^{\varepsilon}\right)$ and the end vertex $t\left(i^{\varepsilon}\right)$, to be defined as

$$
s(i)=l_{i} \alpha, t(i)=r_{i} \alpha, s\left(i^{-1}\right)=r_{i} \alpha, t\left(i^{-1}\right)=l_{i} \alpha .
$$

Recall that the (bi)free group $F^{\mathscr{C}} \mathcal{G}_{1}$ can be realized as the set of all reduced words in the alphabet $I \cup I^{-1}$ together with the empty word 1 . A word $w$ is reduced if it does not contain subwords of the form $i i^{-1}$ or $i^{-1} i, i \in I$. A walk $w$ on the graph $\operatorname{dg}(\alpha)$ is a finite sequence $i_{1} i_{2} \ldots i_{n}$ of edges $i_{k} \in E(d g(\alpha))$ such that $t\left(i_{k}\right)=s\left(i_{k+1}\right)$ for $k=1, \ldots, n-1$. A walk $w$ is admissible if the associated sequence of labels is a reduced word in $I \cup I^{-1}$. For $w=i_{1} \ldots i_{n}$ put $s(w)=s\left(i_{1}\right)$ and $t(w)=t\left(i_{n}\right)$. The trivial walk on $x \alpha$, that is, a walk starting and ending at $x \alpha$ and having no labels will be also called admissible and will be associated with the empty word 1 on the alphabet $I \cup I^{-1}$. The set of all admissible walks on $d g(\alpha)$ will be denoted by $W(\alpha)$.

The free strict inverse semigroup which coincides with the bifree strict inverse semigroup $F \mathscr{S} \mathscr{I}_{I}$ now can be constructed as follows (see [3]).

THEOREM 3.8. Let I be a non-empty set, $Y, X$ etc. be as in Definition 1 and $d g(\alpha)$ be as in Definition 2. Let

$$
S=\left\{(x \alpha, \alpha, g, y \alpha) \mid(x \alpha, \alpha, y \alpha) \in F \mathscr{B}_{l}, g \in W(\alpha) \subseteq F^{\mathscr{G}_{l}}, s(g)=x \alpha, t(g)=y \alpha\right\},
$$

endowed with multiplication

$$
(x \alpha, \alpha, g, y \alpha)(u \beta, \beta, h, v \beta)=(x \delta, \delta, r(g h), v \delta)
$$

where $\delta=\alpha \vee \omega_{\{y, u\}} \vee \beta$ and $r(g h)$ is the reduced form of gh. Let $\phi: i \mapsto$ $\left(l_{i} \varepsilon_{i}, \varepsilon_{i}, i, r_{i} \varepsilon_{i}\right), i^{*} \mapsto\left(r_{i} \varepsilon_{i}, \varepsilon_{i}, i^{-1}, l_{i} \varepsilon_{i}\right)$. Then $(S, \phi)$ is the (bi)free strict inverse semigroup on l.

In the same way as for the combinatorial case the following model of the bifree object $F Y O_{\text {I }}$ can be obtained. All we have to do is to find the subsemigroup $S$ of 
$F \mathscr{B}, \times F \mathscr{R} \mathscr{B}, \times F \mathscr{G}$, which is generated by all elements of the form

$$
\left\{\left(l_{i}, l_{i} \varepsilon_{i}, \varepsilon_{i}, i, r_{i} \varepsilon_{i}, r_{i}\right),\left(r_{i}, r_{i} \varepsilon_{i}, \varepsilon_{i}, i^{-1}, l_{i} \varepsilon_{i}, l_{i}\right) \mid i \in I\right\} .
$$

The proof of the following result can be done in the same way as for the combinatorial case (Theorem 3.5). One has to take into account that $\left(l_{i}, l_{i} \varepsilon_{i}, \varepsilon_{i}, 1, l_{i} \varepsilon_{i}, l_{i}\right)$, $\left(r_{i}, r_{i} \varepsilon_{i}, \varepsilon_{i}, 1, r_{i} \varepsilon_{i}, r_{i}\right) \in S$ for all $i \in I$. Further, from Theorem 4.4 in [7], $\mathscr{S} \mathscr{L} \mathcal{U}=$ $(\mathscr{S} \mathscr{L} \cup \cap \mathscr{I}) \vee(\mathscr{S} \mathscr{L} \mathscr{R} \cap \mathscr{R} \mathscr{B})=\mathscr{S} \mathscr{I} \vee \mathscr{R} \mathscr{Z}=\mathscr{B} \vee \mathscr{G} \vee \mathscr{R} \mathscr{Z}=\mathscr{C} \mathscr{S} \mathscr{U} \vee \mathscr{G}$ and dually for the $\mathscr{R}$-unipotent case.

TheOREM 3.9. Let I be a non-empty set and $Y, X, d g(\alpha)$ etc. be as in Theorem 3.8. Let

$$
S=\left\{(x, \alpha, g, y) \mid(x, \alpha, y) \in F_{\mathscr{C P O}}, g \in W(\alpha) \subseteq F^{G_{l}}, s(g)=x \alpha, t(g)=y \alpha\right\}
$$

endowed with multiplication

$$
(x, \alpha, g, y)(u, \beta, h, v)=\left(x, \alpha \vee \omega_{\{y, u\}} \vee \beta, r(g h), v\right) .
$$

Let $\phi: i \mapsto\left(l_{i}, \varepsilon_{i}, i, r_{i}\right), i^{*} \mapsto\left(r_{i}, \varepsilon_{i}, i^{-1}, l_{i}\right)$. Then $(S, \phi)$ is the bifree strict orthodox semigroup on $I$. The bifree strict $\mathscr{L}$-unipotent semigroup can be obtained by replacing the entry $x$ in $(x, \alpha, g, y)$ by $x \alpha$ and dually for the bifree strict $\mathscr{R}$-unipotent semigroup.

By [7, Theorem 4.4], $\mathscr{L}(\mathscr{S O}) \cong \mathscr{L}(\mathscr{C} \mathscr{S O}) \times \mathscr{L}(\mathscr{G}) \cong \mathscr{L}(\mathscr{B}) \times \mathscr{L}(\mathscr{R} \mathscr{B}) \times \mathscr{L}(\mathscr{G})$. We now describe the fully invariant congruences on $F \mathscr{P O}$.

THEOREM 3.10. Let $\mathscr{A} \in \mathscr{L}(\mathscr{G})$ and $\sigma_{\mathscr{A}}$ denote the fully invariant congruence on $F \mathscr{G}$, such that $F \mathscr{G}_{l} / \sigma_{\mathscr{A}} \cong F \mathscr{A}_{l}$. For $\mathscr{V} \in \mathscr{L}(\mathscr{P O})$ denote by $\rho_{V}$ the fully invariant congruence on $F \mathscr{P O}$, corresponding to $\mathcal{V}$. Let $(x, \alpha, g, y),(u, \beta, h, v) \in F \mathscr{P O}$. Then the following assertions hold.

(1) $(x, \alpha, g, y) \rho_{\mathscr{C S O} \checkmark A}(u, \beta, h, v)$ if and only if $\alpha=\beta, x=u, y=v$ and $g \sigma_{\mathscr{A}} h$.

(2) $(x, \alpha, g, y) \rho_{\mathcal{N B} \vee A A}(u, \beta, h, v)$ if and only if $d \alpha=d \beta, x=u, y=v, g \sigma_{s A} h$.

(3) $(x, \alpha, g, y) \rho_{\mathscr{A B} \vee A}(u, \beta, h, v)$ if and only if $x=u, y=v, g \sigma_{\mathscr{A}} h$.

(4) $(x, \alpha, g, y) \rho_{\mathscr{G S P S Q} q \cup \mathscr{A}}(u, \beta, h, v)$ if and only if $\alpha=\beta, x \alpha=u \beta, y=v, g \sigma_{\mathscr{A}} h$.

(5) $(x, \alpha, g, y) \rho_{\mathscr{A N S A} A}(u, \beta, h, v)$ if and only if $d \alpha=d \beta, y=v, g \sigma_{\mathscr{A}} h$.

(6) $(x, \alpha, g, y) \rho_{\mathscr{R} \mathscr{X} \vee \mathscr{A}}(u, \beta, h, v)$ if and only if $y=v, g \sigma_{\mathscr{A}} h$.

(7) $(x, \alpha, g, y) \rho_{\mathscr{B} \vee A A}(u, \beta, h, v)$ if and only if $\alpha=\beta, x \alpha=u \beta, y \alpha=v \beta, g \sigma_{\mathscr{A}} h$.

(8) $(x, \alpha, g, y) \rho_{\mathscr{P} \vee \mathscr{A}}(u, \beta, h, v)$ if and only if $d \alpha=d \beta, g \sigma_{\mathscr{A}} h$.

(9) $(x, \alpha, g, y) \rho_{\mathscr{A}}(u, \beta, h, v)$ if and only if $g \sigma_{\mathscr{A}} h$.

The $\mathscr{R}$-unipotent congruences are described by the duals of $(4,5,6)$.

Proof. We give a detailed proof only for the case (2). The remaining cases can be proved in the same fashion. We consider the following mappings: $\phi: F \mathscr{Y O} \rightarrow F \mathscr{Y}_{1}$, $\psi: F \mathscr{Y O} \mathcal{O}_{1} \rightarrow F \mathscr{R} \mathscr{B}_{1}$ and $\xi: F \mathscr{Y O} \mathscr{O}_{1} \rightarrow F \mathscr{A}_{1}$, defined by $(x, \alpha, g, y) \phi=c(d \alpha),(x, \alpha, g, y) \psi$ $=(x, y)$ and $(x, \alpha, g, y) \xi=g \sigma_{\mathscr{A}}$. These mappings are the canonical homomorphisms of $F \mathscr{P O}$, onto the relatively bifree objects in $\mathscr{S}, \mathscr{R} \mathscr{B}$ and $\mathscr{A}$, respectively, which induce the congruences $\rho_{\mathscr{S}}, \rho_{\mathscr{R} \mathscr{B}}$ and $\rho_{\mathscr{A}}$, respectively. Since $\rho_{\mathcal{N S B} \mathscr{A}}=\rho_{\mathscr{P} \vee \mathscr{R} \mathscr{B} \vee \mathscr{A}}=\rho_{\mathscr{S}} \cap \rho_{\mathscr{A} \mathscr{B}} \cap \rho_{\mathscr{A}}$ the assertion follows.

Defining an involution $x \mapsto x^{*}$ on $F \mathscr{O O}$, by $(x, \alpha, g, y)^{*}=\left(y, \alpha, g^{-1}, x\right)$ we get a strict orthodox *-semigroup. Similarly, if $\mathscr{A} \in \mathscr{L}(\mathscr{G})$ and $\mathscr{V}$ is any one of the self dual $e$-varieties 
$\mathscr{T}, \mathscr{S}, \mathscr{B}, \mathscr{R} \mathscr{B}, \mathcal{N} \mathscr{B}, \mathscr{C} \mathscr{O}$, then on $F \mathscr{S O} / \rho_{\mathcal{V} \mathscr{A}}$ a $*$-operation can be defined in a canonical way by interchanging the outer entries and inverting the group entry so that the resulting semigroup becomes a strict orthodox *-semigroup. In this case, the congruence $\rho_{\mathscr{V} \vee \mathscr{A}}$ respects the involution. In particular, having shown that $F \mathscr{Y O} O_{l}$, endowed with the mentioned involution, is the free strict orthodox $*$-semigroup then each congruence $\rho_{\vee \vee A}$ defines a variety of strict orthodox *-semigroups. In the next section we shall see that all varieties of strict orthodox *-semigroups are thereby obtained.

4. Varieties of strict orthodox *-semigroups. In this section we shall consider $\mathscr{L}\left(\mathscr{S O} \mathcal{O}^{*}\right)$, the lattice of all strict orthodox *-semigroup varieties. The following important results concerning the $\mathscr{D}$-classes of a regular $*$-semigroup have been obtained by Nordahl and Scheiblich [16].

THEOREM 4.1. Let $S$ be a regular *-semigroup and $a \in S$. Then the mapping $x \mapsto x^{*}$ $\left(x \in R_{a}\right)$ is a bijection between $R_{a}$ and $L_{a^{*}}$ which preserves idempotents and $\mathscr{H}$-classes.

THEOREM 4.2. The involution* on a regular *-semigroup fixes one and only one idempotent per $\mathscr{R}$-class.

The dual assertions for the $\mathscr{L}$-classes of course also hold. Similarly as for the case of the $e$-variety of all strict orthodox semigroups we have the following.

TheOREM 4.3. $\mathscr{S O}^{*}=\mathscr{B}^{*} \vee \mathscr{R} \mathscr{B}^{*} \vee \mathscr{G}^{*}$.

Proof. Let $I$ be a non-empty set. Let $F \mathscr{Y O} \mathcal{O}_{I}$ and $F \mathscr{Y O} \mathcal{O}_{I}^{*}$ denote the bifree strict orthodox and the free strict orthodox *-semigroups, respectively. In the realization of Theorem 3.9, $F Y O_{I}$ is generated as a semigroup by the elements $\left\{\left(l_{i}, \varepsilon_{i}, i, r_{i}\right)\right.$, $\left.\left(r_{i}, \varepsilon_{i}, i^{-1}, l_{i}\right) \mid i \in I\right\}$. As pointed out in Section 3, FPO, admits an involution * $(x, \alpha, g, y) \mapsto\left(y, \alpha, g^{-1}, x\right)$ so that $F \mathscr{S O}$, becomes a strict orthodox *semigroup. Now consider $I$ as a subset of $F \mathscr{S O} \mathcal{O}_{l}^{*}$. Let $f:\left(l_{i}, \varepsilon_{i}, i, r_{i}\right) \mapsto i,\left(r_{i}, \varepsilon_{i}, i^{-1}, l_{i}\right) \mapsto i^{*}$. The mapping $f$ extends uniquely to a homomorphism $f: F \mathscr{P O} \rightarrow F \mathscr{S O} \mathscr{O}_{I}^{*}$ which, in addition, respects involution. On the other hand, since $F \mathscr{O} O_{I}$ is a strict orthodox *-semigroup, the mapping $g: i \mapsto\left(l_{i}, \varepsilon_{i}, i, r_{i}\right)$ extends uniquely to a *homomorphism $g: F \mathscr{O} \mathcal{O}_{I}^{*} \rightarrow F \mathscr{Y O}$. Then $g f: F \mathscr{Y O} O_{l}^{*} \rightarrow F \mathscr{Y O} O_{l}^{*}$ is a $*$-homomorphism satisfying $i g f=i$ for all $i \in I$. Uniqueness of $g f$ implies $g f=\mathrm{id}_{F \mathscr{S O}}$. Dually also $f g=\mathrm{id}_{F \mathscr{S O} O_{l}}$. Consequently, $F \mathscr{S O} \mathscr{O}^{*} \cong F \mathscr{S O} \mathcal{O}_{l}$. Remember that the semigroup $F \mathcal{S O}$, consists of the following elements (in redundant form):

$$
\left\{(x, x \alpha, \alpha, g, y \alpha, y) \mid(x \alpha, \alpha, y \alpha) \in F \mathscr{B}_{l}, g \in W(\alpha) \subseteq F \mathscr{G}_{l}, s(g)=x \alpha, t(g)=y \alpha\right\} .
$$

From this it is obvious that $F \mathscr{S O} \mathcal{O}_{I}^{*} \cong F \mathscr{Y O}$, is a subdirect product of the relatively free

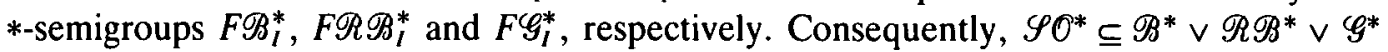
and the converse inclusion is trivial.

Similarly it can be seen that $\mathscr{S O} \mathscr{O}^{*}=\mathscr{C} \mathscr{S O} \mathcal{O}^{*} \vee \mathscr{G}^{*}$ by showing that $\mathscr{C} \mathscr{S} \mathscr{O}^{*}=\mathscr{B}^{*} \vee \mathscr{R} \mathscr{B}^{*}$. The following result can be obtained from [9, Theorem 6].

THEOREM 4.4. Let I be a non-empty set, $P$ be an equivalence relation on $I, G$ be a group and $\mathcal{M}^{0}(I, G ; P)=I \times G \times I \cup\{0\}$ where $0 \notin I \times G \times I$. Define a multiplication on $\mathcal{M}^{0}(I, G ; P)$ by

$$
\begin{gathered}
(i, g, j)(k, h, l)=\left\{\begin{array}{cc}
(i, g h, l) & \text { if }(j, k) \in P \\
0 & \text { if }(j, k) \notin P,
\end{array}\right. \\
0(i, g, j)=(i, g, j) 0=00=0,
\end{gathered}
$$


and an involution by

$$
(i, g, j)^{*}=\left(j, g^{-1}, i\right), 0^{*}=0 .
$$

Then $\mathcal{M}^{0}(I, G ; P)$ is a completely 0 -simple orthodox *-semigroup. Conversely, every completely 0 -simple orthodox $*$-semigroup $(\neq\{0\})$ can be so constructed.

Let $A$ and $B$ be two completely (0-)simple semigroups. Let $I=\left(A \times\left\{0_{B}\right\}\right) \cup\left(\left\{0_{A}\right\} \times\right.$ $B) \subseteq A \times B$. Here $0_{A}$ and $0_{B}$ denote the zeros of $A$ and $B$, respectively, provided these zeros exist. Otherwise the respective set is taken to be empty. The semigroup $A \otimes B=A \times B / I$ is completely (0-)simple. A Rees matrix representation of $A \otimes B$ by means of the respective representations of $A$ and $B$ can be obtained easily (see [7]). Let $S=\mathcal{M}^{0}(I, G ; P)$ be a completely 0 -simple orthodox $*$-semigroup. Then $\mathcal{M}^{0}(I ; P)=I \times I \cup$ $\{0\}$, equipped with multiplication

$$
\begin{gathered}
(i, j)(k, l)=\left\{\begin{array}{cc}
(i, l) & \text { if }(j, k) \in P \\
0 & \text { if }(j, k) \notin P,
\end{array}\right. \\
0(i, j)=(i, j) 0=00=0,
\end{gathered}
$$

and involution

$$
(i, j)^{*}=(j, i), 0^{*}=0,
$$

is the combinatorial part of $\mathcal{M}^{0}(I, G ; P)$.

By Theorem $4.4, S \cong \mathscr{M}^{0}(I, G ; P) \cong \mathcal{M}^{0}(I ; P) \otimes G$ for each completely 0 -simple orthodox *-semigroup $S$. Denote by $B(I / P)$ the combinatorial Brandt semigroup on $I / P \times I / P \cup\{0\}$ and let $R B(I)$ be the rectangular $*$-band on $I \times I$. The semigroup $B(I / P) \otimes R B(I)$ can be realized as follows:

$$
B(I / P) \otimes R B(I)=I / P \times I \times I \times I / P \cup\{0\},
$$

endowed with multiplication

$$
(i P, j, k, l P)\left(i^{\prime} P, j^{\prime}, k^{\prime}, l^{\prime} P\right)=\left\{\begin{array}{cc}
\left(i P, j, k^{\prime}, l^{\prime} P\right) & \text { if }\left(l, i^{\prime}\right) \in P \\
0 & \text { if }\left(l, i^{\prime}\right) \notin P,
\end{array}\right.
$$

and all other products are taken to be zero, and also endowed with involution

$$
(i P, j, k, l P)^{*}=(l P, k, j, i P), 0^{*}=0 .
$$

The mapping $\phi: \mathcal{M}^{0}(I ; P) \rightarrow B(I / P) \otimes R B(I)$, defined by $(i, j) \mapsto(i P, i, j, j P), 0 \mapsto 0$ is an injective *-homomorphism. In particular, $\mathcal{M}^{0}(I ; P)$ is isomorphic to a $*$-subsemigroup of $B(I / P) \otimes R B(I)$. We have thus proved the following. Given a completely 0 -simple orthodox *-semigroup $S$ with maximal subgroup $G$, then $S \in\langle G\rangle^{*} \vee \mathscr{B}^{*} \vee \mathscr{R} \mathscr{B} *$. Further, $S \in\langle G\rangle^{*} \vee \mathscr{B}^{*}$ if and only if $S$ contains no non-trivial rectangular band, that is, if and only if $S$ is inverse. Also, $S \in\langle G\rangle^{*} \vee \mathscr{S}^{*} \vee \mathscr{R}_{\mathscr{B}^{*}}$ if and only if $S$ contains no $\mathscr{H}$-class which is null. Precisely in this case, $P$ is the universal relation, or equivalently, $S$ is a rectangular (*-)group with a zero adjoined.

LEMMA 4.5. Let $S$ be a strict regular *-semigroup and let $S=\left(X ; I_{\alpha}, f_{\alpha, \beta}\right)$ be represented as in Theorem 2.1. Then the partial homomorphisms $f_{\alpha, \beta}$ respect the involution. 
Proof. Let $\alpha \geq \beta \in X$ and $x \in I_{\alpha}^{*}$. Then by construction, $\left(x f_{\alpha, \beta}\right)\left(x f_{\alpha, \beta}\right)^{*}=x\left(x f_{\alpha, \beta}\right)^{*}$ and $\left(x f_{\alpha, \beta}\right) x^{*}=\left(x f_{\alpha, \beta}\right)\left(x^{*} f_{\alpha, \beta}\right)$. Since $\left(x f_{\alpha, \beta}\right)\left(x f_{\alpha, \beta}\right)^{*}$ is fixed under the involution, we have $\left(x f_{\alpha, \beta}\right)\left(x f_{\alpha, \beta}\right)^{*}=x\left(x f_{\alpha, \beta}\right)^{*}=\left[x\left(x f_{\alpha, \beta}\right)^{*}\right]^{*}=\left(x f_{\alpha, \beta}\right) x^{*}=\left(x f_{\alpha, \beta}\right)\left(x^{*} f_{\alpha, \beta}\right)=\left(x x^{*}\right) f_{\alpha, \beta}$. In particular, $\left[\left(x x^{*}\right) f_{\alpha, \beta}\right]^{*}=\left(x x^{*}\right) f_{\alpha, \beta}$. Now we have $\left(x f_{\alpha, \beta}\right)^{*}=\left[\left(x x^{*}\right) f_{\alpha, \beta}\left(x f_{\alpha, \beta}\right)\right]^{*}=$ $\left[\left(x x^{*}\right) f_{\alpha, \beta} x\right]^{*}=x^{*}\left[\left(x x^{*}\right) f_{\alpha, \beta}\right]^{*}=x^{*}\left(x x^{*}\right) f_{\alpha, \beta}=\left(x^{*} f_{\alpha, \beta}\right)\left(x x^{*}\right) f_{\alpha, \beta}=x^{*} f_{\alpha, \beta}$.

Corollary 4.6. Let $\mathscr{V}^{*} \in \mathscr{L}\left(\mathscr{9 O ^ { * } )}\right.$ be a variety of strict orthodox *-semigroups. Then

$$
\mathscr{V}^{*}=\left(\mathscr{V}^{*} \cap \mathscr{G}^{*}\right) \vee\left(\mathscr{V}^{*} \cap \mathscr{B}^{*}\right) \vee\left(\mathscr{V}^{*} \cap \mathscr{R} \mathscr{B}^{*}\right)
$$

Proof. Let $\mathscr{V}^{*} \in \mathscr{L}\left(\mathscr{P O} \mathscr{O}^{*}\right)$ and $\mathscr{S} \in \mathscr{V}^{*}$. Each $\mathscr{D}$-class of $S=\left(X ; I_{\alpha}, f_{\alpha, \beta}\right)$ is invariant under the involution. Hence each principal factor $I_{\alpha}$ of $S$ in fact is a completely (0-)simple orthodox *-semigroup contained in $\mathscr{V}^{*}$. As shown above, $I_{\alpha} \in\left(\mathscr{V}^{*} \cap \mathscr{G}^{*}\right) \vee\left(\mathscr{V}^{*} \cap \mathscr{B}^{*}\right) \vee$ $\left(\mathscr{V}^{*} \cap \mathscr{R}_{\mathscr{B}^{*}}\right)$ for each $I_{\alpha}$. By [3, Theorem 2.3], $S$ is a subdirect product of the semigroups $I_{\alpha}$. Since the mappings $f_{\alpha, \beta}$ respect the involution, by the proof of [3, Theorem 2.3], $S$ is also isomorphic to a *-subsemigroup of the direct product of its principal factors. Consequently, $S \in\left(\mathscr{V}^{*} \cap \mathscr{G}^{*}\right) \vee\left(\mathscr{V}^{*} \cap \mathscr{B}^{*}\right) \vee\left(\mathscr{V}^{*} \cap \mathscr{R} \mathscr{B}^{*}\right)$.

This yields a decomposition of the lattice $\mathscr{L}\left(\mathscr{Y} \mathcal{O}^{*}\right)$. Taking into account that $\left(\mathcal{U}^{*}, \mathscr{V}^{*}, \mathscr{W}^{*}\right) \mapsto \mathscr{U}^{*} \vee \mathscr{V}^{*} \vee \mathscr{W}^{*}$ is also an injective mapping of $\mathscr{L}\left(\mathscr{G}^{*}\right) \times \mathscr{L}\left(\mathscr{B}^{*}\right) \times$ $\mathscr{L}\left(\mathscr{R} \mathscr{B}^{*}\right)$ into $\mathscr{L}\left(\mathscr{S O ^ { * }}\right)$ then in fact we have obtained the following.

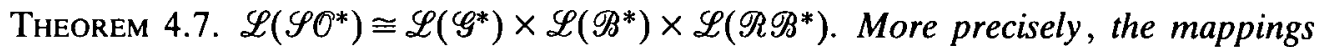
$\mathscr{V}^{*} \mapsto\left(\mathscr{V} \cap \mathscr{G}^{*}, \mathscr{V}^{*} \cap \mathscr{B}^{*}, \mathscr{V}^{*} \cap \mathscr{R} \mathscr{B}^{*}\right)$ and $\left(\mathscr{U}^{*}, \mathscr{V}^{*}, \mathscr{W}^{*}\right) \mapsto \mathscr{U}^{*} \vee \mathscr{V}^{*} \vee \mathscr{W}^{*}$ are mutual inverse isomorphisms between $\mathscr{L}\left(\mathscr{S} \mathscr{O}^{*}\right)$ and $\mathscr{L}\left(\mathscr{G}^{*}\right) \times \mathscr{L}\left(\mathscr{B}^{*}\right) \times \mathscr{L}\left(\mathscr{R} \mathscr{B}^{*}\right)$.

At variance with the case of the e-variety $\mathscr{R} \mathscr{B}$ or the completely regular variety $\mathscr{R} \mathscr{B}^{-1}, \mathscr{L}\left(\mathscr{R} \mathscr{B}^{*}\right)$ is the two element chain rather than the four element diamond lattice (see for instance Adair [1]). Each variety of strict orthodox *-semigroups now corresponds to a self dual $e$-variety of strict orthodox semigroups. Also, the relatively free objects in the varieties of strict orthodox *-semigroups coincide with the bifree objects in the associated $e$-varieties. But the bifree objects in the $\mathscr{L}$ - and $\mathscr{R}$-unipotent $e$-varieties which are not inverse do not have an interpretation as relatively free strict orthodox *-semigroups.

Finally we give a basis of identities for the varieties $\mathscr{S O ^ { * }}$ and $\mathscr{C} \mathscr{S} 0^{*}$.

THEOREM 4.8. The unary semigroup variety $\mathcal{S O}^{*}$ is defined by the following laws.

(1) $(x y) z=x(y z)$

(2) $\left(x^{*}\right)^{*}=x$

(3) $(x y)^{*}=y^{*} x^{*}$

(4) $x x^{*} x=x$

(5) $x x^{*} y y^{*} z z^{*}=\left(x x^{*} y y^{*} z z^{*}\right)^{2}$

(6) $x y x^{*}\left(x y x^{*}\right)^{*}=\left(x y x^{*}\right)^{*} x y x^{*}$

Addition of the law

(7) $x y x^{*}=\left(x y x^{*}\right)^{2}$

yields a basis of identities for $\mathscr{C S O}^{*}$. In this case, (6) may be replaced by

(6') $x y x^{*}=\left(x y x^{*}\right)^{*}$.

Proof. By Theorems 3.7, 3.9 and 4.3 the laws (1-6) hold in every strict orthodox *-semigroup. Conversely, let $S$ be a unary semigroup satisfying (1-6). By Theorem $2.4, S$ is orthodox. Hall [6] has pointed out that a regular semigroup $S$ is strict if and only if each local submonoid $e S e, e \in E(S)$, is a semilattice of groups. Let $e \in E(S)$. The regular 
semigroup $e S e$ is isomorphic (as a semigroup) to the regular semigroup $e e^{*} \mathrm{See}^{*}=e S e^{*}$ via the mapping $x \mapsto x e^{*}$. Further, $e S e^{*}$ is invariant under the involution *. In particular, $e S e^{*}$ is a regular *-semigroup which by (6) satisfies the identity $x^{*} x=x x^{*}$. A fortiori, $e S e^{*}$ satisfies the law $x x^{*} x^{*} x=x^{*} x x x^{*}$ so that $e S e^{*}$ is an inverse semigroup with inversion $x \mapsto x^{*}$. The identity $x x^{*}=x^{*} x$ then implies that $e S e^{*}$ is a semilattice of groups. By Theorem 3.7 each combinatorial strict orthodox $*$-semigroup satisfies (7) and $\left(6^{\prime}\right)$. On the other hand, (7) implies that the Clifford semigroups $e S e^{*}$ are inverse semigroups satisfying the law $x=x^{2}$. Consequently, as above, each local submonoid $e S e$ is a semilattice and thus $S$ is a combinatorial strict regular semigroup.

\section{REFERENCES}

1. C. L. Adair, Bands with involution, J. Algebra 75 (1982), 297-314.

2. K. Auinger, Free strict inverse semigroups, J. Algebra (to appear).

3. K. Auinger, Free objects in joins of strict inverse and completely simple semigroup varieties, J. London Math. Soc. (to appear).

4. G. Grätzer, Universal Algebra (Van Nostrand, Princeton, 1978).

5. G. Grätzer, General Lattice Theory (Birkhäuser, Basel, 1978).

6. T. E. Hall, Identities for existence varieties of regular semigroups, Bull. Austral. Math. Soc. 40 (1989), 59-77.

7. T. E. Hall, Regular semigroups: amalgamation and the lattice of existence varieties, Algebra Universalis 28 (1991), 79-108.

8. T. E. Hall, A concept of variety for regular semigroups; in Semigroup Theory, Proceedings of the Monash University Conference on Semigroup Theory in Honor of G. B. Preston, eds. T. E. Hall, J. C. Meakin and P. R. Jones (World Scientific, Singapore, 1991), 101-116.

9. T. E. Hall, On regular semigroups whose idempotents form a subsemigroup, Bull. Austral. Math. Soc. 1 (1969), 195-208.

10. F. Harary, Graph Theory (Addison-Wesley, 1971).

11. J. M. Howie, An Introduction to Semigroup Theory. (Academic Press, London, 1976).

12. J. Kadourek and M. B. Szendrei, A new approach in the theory of orthodox semigroups, Semigroup Forum 40 (1990), 257-296.

13. G. Lallement, Demi-groupes réguliers, Ann. Math. Pura Appl. 77 (1967), 47-129.

14. G. Lallement and M. Petrich, Structure d'une classe de demi-groupes réguliers, J. Math. Pures Appl. 48 (1969), 345-397.

15. S. W. Margolis, J. C. Meakin and J. B. Stephen, Free objects in certain varieties of inverse semigroups, Canad. J. Math. 42 (1990), 1084-1097. 369-377.

17. M. Petrich, Regular semigroups satisfying certain conditions on idempotents and ideals, Trans. Amer. Math. Soc. 170 (1972), 245-267.

18. M. Petrich, Inverse Semigroups (Wiley, New York, 1984).

19. N. R. Reilly, Free combinatorial strict inverse semigroups, J. London Math. Soc. (2) 39 (1989), 102-120.

20. M. B. Szendrei, Free *-orthodox semigroups, Simon Stevin 59 (1985), 175-201

21. M. B. Szendrei, A new interpretation of free orthodox and generalized inverse *semigroups; in Semigroups, Theory and Applications, Proceedings of the 1986 Oberwolfach Conference on Semigroups, eds. G. Lallement, H. Jürgensen and H. Weinert, Lecture Notes in Mathematics 1320 (Springer-Verlag, 1988), 358-371.

22. Y. T. Yeh, The existence of $e$-free objects in $e$-varieties of regular semigroups, Int. J. Alg. Comput. (to appear).

INSTITUT FÜR MATHEMATIK, STRUDLHOFGASSE 4, A-1090 WIEN. 